\title{
Design and Fabrication of Integrated Microchannel and Peristaltic Micropump System for Inertial Particle Separation
}

\author{
Utku Sönmez, Muhammed Bekin and Levent Trabzon \\ Department of Mechanical Engineering, Istanbul Technical University, Istanbul, Turkey \\ MEMS Research Center (www.mems.itu.edu.tr)
}

\begin{abstract}
In particle separation applications, conventional syringe pumps are widely used to supply fluid flow into microchannels at a controlled flow rate. However, their bulky structures lack the development of compact particle separation systems which is essential for all LoC (Lab on a Chip) systems. In this study, we designed and fabricated a peristaltic micropump which can be integrated into an inertial particle separation microchannel at the same layer with a compact design. Since inertial particle separation can be done without a need for an external force field, we aimed to develop a $\mu$ TAS (Micro Total Analysis Systems) system which is able to realize particle separation in an integrated micropump-microchannel system. The circular micropump channel made of two PDMS layers and its width is optimized. The 3D-Printed micropump is actuated by a stepper motor, and the rate of pumped fluid is monitored by an LCD screen connected and programmed to system according to the system parameters. Micropump has a theoretical capacity of supplying particle carrying fluid at the flow rate of $25.47 \mathrm{ml} / \mathrm{min}$ when the stepper motor is rotated at 330 rpm.
\end{abstract}

\section{Introduction}

Micropumps are widely used to produce microscale fluid flows for the application fields ranging from biological and chemical assays like drug delivery systems, micro-dosing control mechanisms and particle separation devices to aerospace and microelectronics industry [1]. There is plenty of micropump designs that can be found in literature dedicated to carry out different tasks [2]. They are generally classified with respect to the working principles of their actuator mechanisms. From this point of view, SMA (Sheet Metal Alloy) actuated micropumps [3], piezoelectric micropumps [4], ICPF (Ionic Conductive Polymer Film) based micropumps [5], peristaltic micropumps [6] and MHD (magnetohydrodynamic) micropumps [7] are developed in the last decade for variety of applications. SMA actuated micropumps, piezoelectric micropumps, and ICPF micropumps contain a deformable diaphragm, which pumps the fluid to a certain direction with the aid of one-way valves. However, the maximum flow rate that they can supply is not sufficient for applications like inertial particle separation which requires extraordinarily high flow rates [8] since the maximum achievable frequency limit for their membranes is not high enough. On the other hand, they lack on biocompatibility either due to the high electric voltage need to be used, or the materials required to be employed [9]. In this study, we aimed to develop a compact micropump system that can be used for inertial particle separation applications, and integrate it to the Sunflower microchannel geometry that we developed before.

\section{Micropump design}

The micropump that would be suitable for the inertial particle separation should supply particle carrying fluids with relatively high flow rates without fluctuations, and must be integrated to another microchannel geometry in which inertial particle separation will be realized. Furthermore, the mechanism should not be harmful for the biological cells (e.g. high voltage, high working temperature, physical impact, high accelerations) to not to affect their viability to allow post processing of the separated cells. Given these conditions, peristaltic micropumps are the most appropriate type of micropump since they meet all these conditions with a rather simple design and low cost. In literature, there are two similar configurations of peristaltic micropumps which can be integrated to an inertial separation microchannels. Both of these positive displacement pump configurations trap a determined amount of fluid body between two restrictive elements, and force it to move together with them to a certain direction as they displace. The first configuration uses magnetically attracted metal balls that squeeze the deformable micropump channels and sweep them by means of rotating magnets that are connected to a motor [10]. The second configuration, uses rotating ball bearings that are directly 
connected to the motor, and creates the squeezing effect by the preload applied on the micropump channel. The latter one has a line contact between the roller bearings and the deformable PDMS surface instead of a point contact, which in turn deforms the PDMS layer in a more uniform trend, and closes the gaps formed near the microchannel side walls (Figure 1). This reduces the backflow significantly, allowing the micropump to pump the fluid at higher flow rates for the given rotational speed. On the other hand, metal balls in the first configuration are fixed to their circular orbit by means of magnetic forces. It is reported that the metal balls draw apart from their circular orbit and spread away randomly at higher rotational speeds due to the increasing centripetal acceleration acting on them, in a way that the mechanism loses its function [11]. In the rotating ball bearing configuration, this problem is not observed since the rotating ball bearings are fixed to their circular orbit by means of rigid bars. Therefore, the maximum achievable rotational speed of the micropump, thus the maximum achievable flow rate, only depends on the maximum rotational speed of the motor that is used to rotate the ball bearings. Apart from that, the width of the micropump channel should not exceed the width of the squeezed cross section to have proper peristaltic effect. At the same time, the amount of fluid that can be pumped in one revolution linearly increases by the increasing channel width. In the rotating metal balls configuration, the diameter of the balls can be increased to work with wider micropump channels to have higher flow rates.

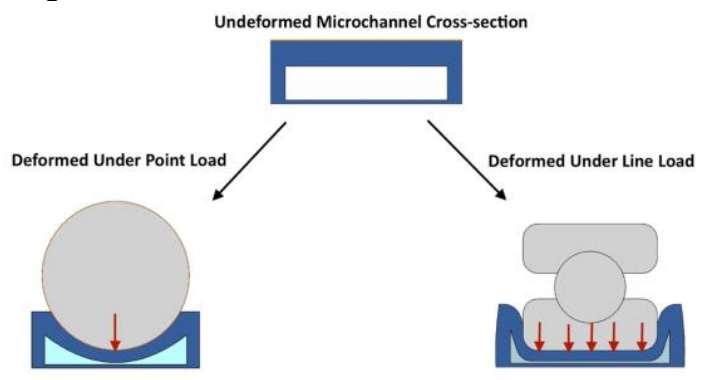

Figure 1. The differences between point load applied by metal balls and the line load applied by the roller bearing itself, in terms of the ability to clog the gap in a uniform manner.

However, in this case, maximum achievable rotational speed decreases since the centripetal acceleration acting on the metal balls increases for the given rotational speed and leads to the breakdown of the mechanism due to the reason described above. In the rotating ball-bearings design, the width of the micropump channel can be tailored freely since there is plenty of ball-bearing with different widths in the market, and the centripetal acceleration acting on the ball bearing is balanced by the rod connected to ball-bearings. Therefore, the fluid pumping capacity of the micropump can be increased tremendously by optimizing the micropump channel width. Due to the advantages described above, we decided to use the peristaltic micropump configuration with rotating ball bearings in our integrated microchannel-micropump system. The concept design described by Zhang et al. [12] for this configuration was used to pump the fluid between $\mathrm{nl} / \mathrm{min}$ and $\mu \mathrm{l} / \mathrm{min}$ range. To meet our system requirements this mechanism was modified to have volumetric flow rates at the range of $\mathrm{ml} / \mathrm{min}$ with less pulsation. Also, it is integrated to a microchannel geometry that we developed in which passive particle separation occurs by means of inertial lift forces and Dean forces. In the following sections, details regarding to the design and the fabrication of such a micropump system is given together with its integration to cell-separating microchannel in a way that it forms a versatile microfluidic platform.

\subsection{Micropump Channel Design}

After examining various micropumps and selecting the most appropriate micropump configuration for our application, we carried out further analysis for the determination of the optimum design variables of the selected peristaltic micropump configuration. The micropump is designed to be manufactured at the same layer width of the Sunflower microchannel [13]. Due to the soft lithography technique that is used to fabricate the microchannel layer of the micropump, it is obligatory for the microchannel and the micropump channel to have the same height. Since the optimum Sunflower-microchannel height is determined to be $60 \mu \mathrm{m}$, we used this value as the height of the micropump channel. On the other hand, the width of the micropump channel can be tailored to increase the amount of fluid pumped per revolution. However, in previous works, various researchers stated that at very high aspect ratios (i.e., width $>>$ height) the roof of the microchannel tends to collapse during the oxygen plasma bonding step [14] [15], sticking on the bottom layer permanently, which, in turn, prevents the fluid partially from flowing through the micropump channel. To find the maximum micropump channel width that can be manufactured without roof collapse, we prepared various micropump channel samples with different channel widths ranging from $2 \mathrm{~mm}$ to $5.5 \mathrm{~mm}$ on a single wafer (Figure 2).

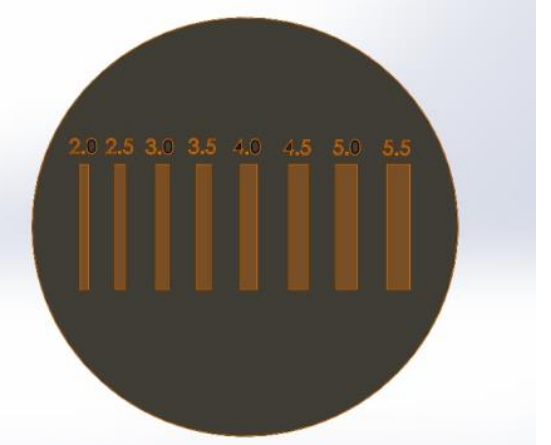

Figure 2. Micropump channel samples with different channel widths are prepared on a single wafer to investigate the maximum channel width that can be achieved without PDMS roof collapse.

We detected that at the width of $5.5 \mathrm{~mm}$, PDMS starts to collapse down and clog the micropump channel permanently (Figure 3 ). Given the experimental results, we decided to have a micropump channel width of $4.5 \mathrm{~mm}$ to be on the safe side. 


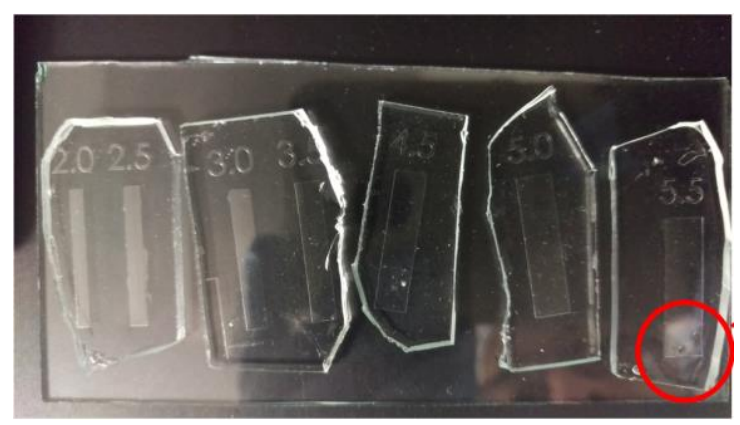

Figure 3. As the channel aspect ratio increases, the roof of the PDMS channel tends to collapse. Experiments done using channel samples with different channel widths show that roof starts to collapse (marked area with red circle) at channel width of $5.5 \mathrm{~mm}$ for the given channel height of $70 \mu \mathrm{m}$.

\subsection{Rotating Bearing Bar and the Ball Bearings}

The number of rods was selected to be 3 to further decrease the pulsation, and the length of each was set to $40 \mathrm{~mm}$ which is the radius of curvature of the micropump channel. The angle between the rods was set to $120^{\circ}$ in such a way that the volume of fluid trapped between each rod is the same. The width of the ball bearings was selected to be $5 \mathrm{~mm}$, which is sufficient to squeeze the micropump channel entirely. Ball bearings were selected from the catalogue of the bearing manufacturer BRC (Washington, United States). Between the ball bearings which have the width of $5 \mathrm{~mm}$, the one which has the smallest outer diameter $\left(\mathrm{d}_{\mathrm{o}}=11 \mathrm{~mm}\right)$ was selected to have a circular motion without locking. The rods were designed to have a decreasing diameter in two steps starting from $8 \mathrm{~mm}$ at the centre, to $4 \mathrm{~mm}$ at the edge, which is the inner diameter of the selected ball bearings. We added the stepped decrement of the diameter to ensure that the ball bearing can still rotate around their own axis freely after the assembly and the rods are deflected less for the given bending force, which rises due to the force applied on the ball bearing while squeezing the micropump channel. Furthermore, we designed the rods with a circular hollow at the centre to nail steel wires, which would further increase their strength against bending moment (Figure 4). Due to the intrinsic properties of the microchannel that are described in the first section of the paper, it only functions well if it is designed to separate particular particles, which means that different PDMS microchannels should be produced to carry out different separation tasks. Furthermore, PDMS microchannel should be disposable for biological applications to eliminate the risk of cross-contamination. For this reason, we designed our micropump in such a way that the PDMS layer can be easily removed and a new one can be replaced without affecting the components of the micropump. Therefore, a hole with $4 \mathrm{~mm}$ diameter drilled at the centre of the bars to connect them to the axis of the motor by means of a screw and a nut, which can be removed and reassembled to replace the microchannel layer. This assembly configuration is also crucial to create the axial force, which would push the ball bearings along the micropump channel.

\subsection{Sunflower Microchannel and Micropump Channel Integration}

The micropump channel and Sunflower microchannel which will be at the same PDMS layer should be manufactured together in the same pattern to accomplish the particle separation task. The pump channel is located around the Sunflower microchannel in such a way that the outlet of the micropump channel is the inlet of the Sunflower microchannel. Since the maximum radius of curvature of the Sunflower microchannel is $2.5 \mathrm{~mm}$, the radius of curvature of the pump channel is selected to be $4 \mathrm{~mm}$ which is sufficiently high to allow the roller bearings to turn about the microchannel, and not excessively high to increase the footprint of the integrated system. Furthermore, two serial reservoirs are located between the inlet of the Sunflower microchannel and the outlet of the micropump channel to decrease the fluctuations intrinsically occurring in peristaltic pumps. Consequently, the integrated form of microchannel-micropump channel is established, as shown in Figure 4. PDMS will be cut. It does not play any functional or structural role in the system. By adding the roller bearings and the bearing rod on top of the system, and rotating them via an electric motor, the final configuration of the integration is obtained.

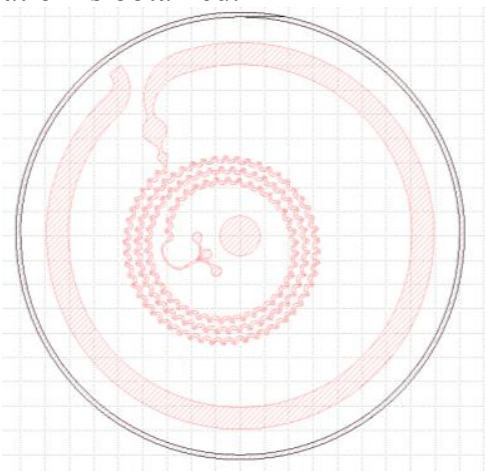

Figure 4. The integrated micropump channel and the Sunflower microchannel with two subsequent reservoirs.

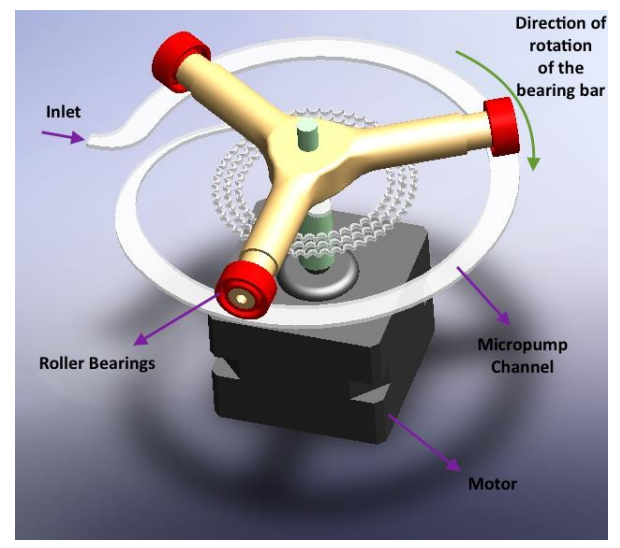

Figure 5. The integrated micropump and microchannel system.

\subsection{Micropump Case}

Micropump case is designed to carry the microchannel and micropump channel, and to house all the electronics used in the mechanism. Furthermore, inlet and outlet tanks are located into the micropump case by means of a movable 
door, which allows us to fill them with the suspension that contains mixed particles, and to collect the fluid from the outlet of the microchannel, which contains separated particles. Plus, a housing for the motor is located at the centre of the case, and other required housings for power switch, LCD monitor and Arduino exits are located with proper dimensions. The case is then designed to be mounted to a bottom plate, which is fixed to the case with screws. The final design of the micropump case can be seen in Figure 6 .

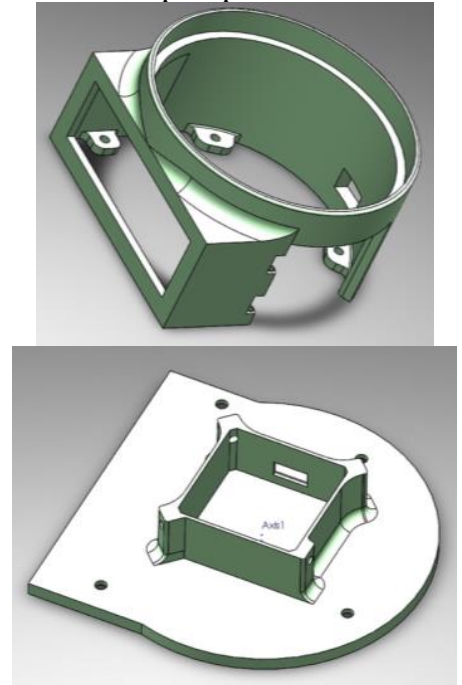

Figure 6. The micropump case (left) and bottom base (right) designed to be able to contain all the electronic and mechanic parts necessary for the system.

Further, inlet and outlet tanks are located on the movable gate on one side of the micropump case. The inlet tank is designed to have a higher inner volume than the outlet tanks since fluid will be separated into two at the exit of the microchannel. The inlet has a $9 \mathrm{ml}$ inner volume which enables us to use the micropump at the optimum flow rate of the Sunflower microchannels for 6 minutes, which is sufficiently high for biological assays, since the specimen volume is much smaller under normal circumstances. Also, a small hole with a diameter of $1.8 \mathrm{~mm}$, which is the diameter of the tygon tubings that we used, is located at the bottom of the inlet tank to maintain a continuous fluid flow from the inlet tank to the micropump channel.

\subsection{Motor and Control Mechanism}

Stepper motor was selected to be used to create the necessary rotational movement since it is easy to control it precisely. For this purpose, Nema-14 stepper motor (National Electrical Manufacturers Association, Virginia-USA) was purchased. The selected stepper motor has 200 steps per revolution which corresponds to $1.8^{\circ}$ of step angle that is sufficient to eliminate fluctuations which may rise due to the discontinuous rotation of the stepper motor. Also, it has $7.4 \mathrm{~V}$ of operating voltage that is also suitable for our application. To drive the stepper motor, EasyDriver ROB-12779 (SparkFun Electronics, Colorado-USA) stepper motor driver was purchased. Furthermore, a 16x2 IIC/I2C/TWI serial LCD display (Surenoo Technology, Guangdong, China) was purchased and integrated to the stepper motor, then it was programmed to show the volumetric flow rate that is pumped by the micropump to the user. Moreover, Arduino Nano microcontroller was purchased to control and program the stepper motor driver and the LCD display via a computer. Lastly, an on/off switch is acquired and connected between the $9 \mathrm{~V}$ Battery power source and the stepper motor to start and shut down the system. All the electronic equipment is connected to each other by using jumper wires.

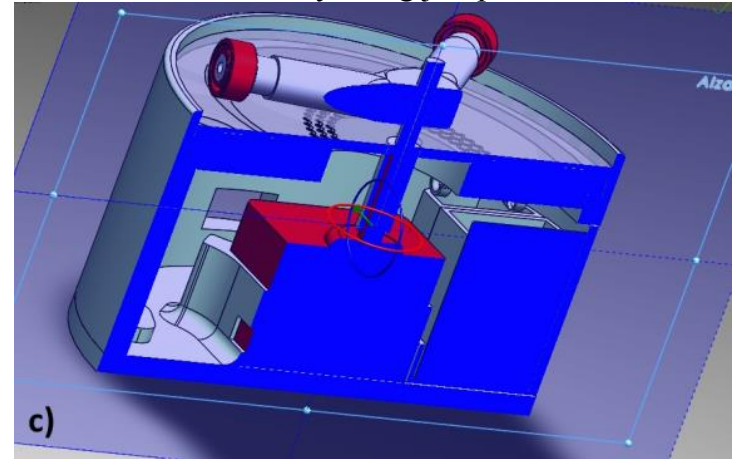

Figure 7. The cross-section of the system without the electronic equipment, wirings and tubing.

\section{Fabrication of Micropump}

Micropump base, micropump case, bottom plate and Y-shaped bearing bar were manufactured by a Ultimaker-2 3D Printer (Geldermalsen-Netherlands) from the CAD documents produced in Solidworks (Figure 8). All the parts were produced by using a PLA filament with a tolerance of $0.2 \mathrm{~mm}$.

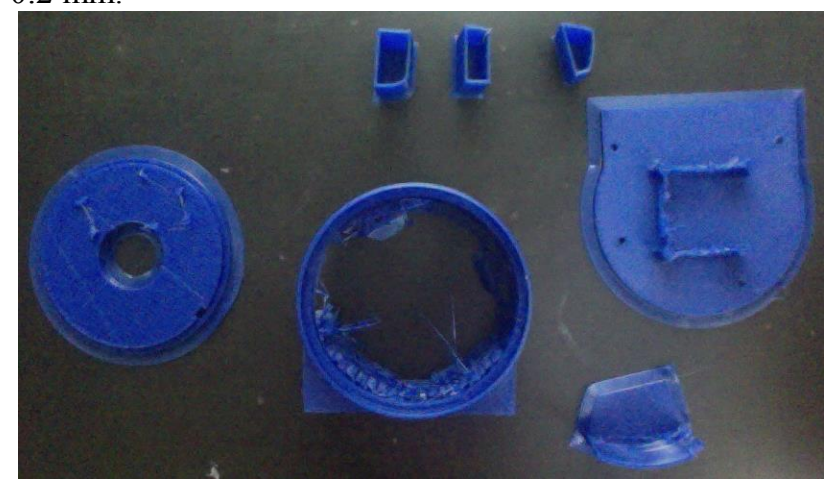

Figure 8. Micropump parts produced by 3D Printer before the assembly.

Supporting parts used by the 3D Printer were then removed by using appropriate tools, and fine-tuning of the parts was done via a sandpaper disk. Three roller bearings (685 Z, BRC Bearing Company) were close fitted to the edges of the bearing bar. The step motor is placed in its housing on the bottom plate and fixed by screws to its centre.

The PDMS layer of the microseparation system that is composed of micropump channel and Sunflower microchannel is first constructed by using CAD software, Solidworks. Then, the CAD geometry is transferred to Heidelberg DWL-66FS laser writer (Heidelberg, Germany) for the mask fabrication. A chrome mask was produced by using DWL. Before the photolithography process, single-side polished Si-wafer was cleansed with Deionized (DI) water and dried with $\mathrm{N} 2$ gas to remove any deposits 
that might have been on the surface. This was followed by spreading Su-8 3000 negative photoresist on the Si-wafer. The PR spreading process was done by spin coating in a Laurell WS 400 B Spinner (North Wales, USA). By using the the Su- 8000 datasheet from MicroChem, necessary process information is found and the necessary rotational speed for the desired photoresist layer thickness is provided. Later on, soft baking is done for 45 minutes at $95^{\circ} \mathrm{C}$ on a hotplate (Prazitherm, Düsseldorf, Germany). Then, using the previously prepared mask carrying the desired pattern, the $\mathrm{Su}-8$ coated $\mathrm{Si}$-wafer was subjected to contact lithography under UV light, in an OAI Model 200 Mask Aligner (San Jose, USA). Sequentially, a post-exposure bake was performed by putting the Si-wafer back on the hot plate on two levels to ensure the solidification of the parts affected by the UV light. The Si-wafer was then immersed in a developer (1-methoxy-2- propanol acetate) medium for 12-15 minutes in which the soluble regions of the PR would dissolve. The wafer was later rinsed with isopropanol and DI water, and dried with N2 gas. After the fabrication process was done, the master template was characterized under the microscope to check for any errors that would cause structural failure in the microchannel manufacturing. After preparing the master template, the PDMS replica molding process started with preparing the PDMS (Sylgard 184, Dow Corning) by mixing it with a curing agent at a weight ratio of 10:1. The pre-polymer was then degassed in a vacuum chamber (Sheldon Manufacturing, Inc.) to remove air bubbles present within the homogeneous mixture. After all bubbles were released, the PDMS prepolymer was slowly poured on the master template, and baked at $90^{\circ} \mathrm{C}$ for more than 3 hours to solidify. The PDMS was later detached from the master template, and fitting holes were punched into the PDMS mould with a $2 \mathrm{~mm}$ biopsy punch. Afterwards, the detached PDMS layer and another PDMS layer that has no pattern on it, were placed inside a Harrick Plasma Cleaner (New York, USA) for the plasma bonding process. After the plasma bonding process inlet and outlet tubings are fit into the punched areas, and the whole PDMS layer is located under the bearing bar.

\section{Results and Discussion}

After the fabrication of each part and the integration of the electronics, assembly of the micropump is done (Figure 10). Given the defined micropump channel dimensions, this peristaltic micropump has the capacity to pump $79 \mathrm{~mm}^{3}$ of fluid per revolution into the microchannel. Under the given loads on the stepper motor, we experimentally found that it is possible to reach $330 \mathrm{rpm}$ by reducing the micro delay of the stepper motor down to $60 \mu \mathrm{s}$.

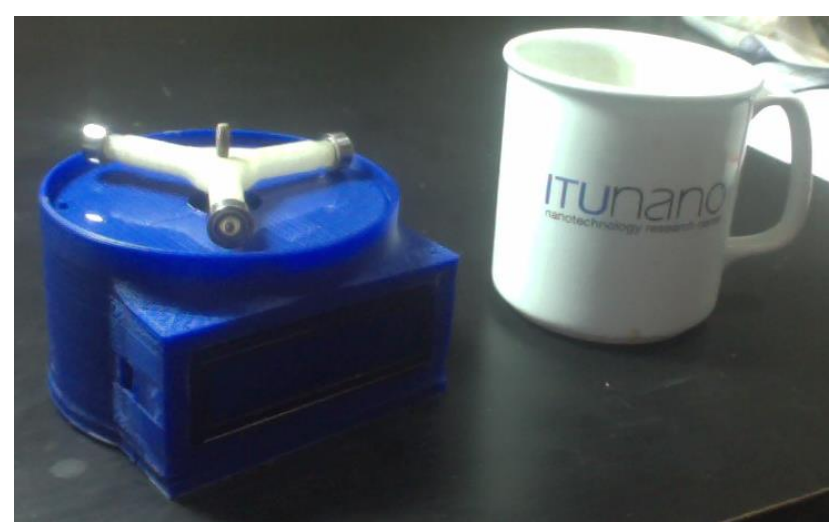

Figure 9. The final form of the micropump with a coffee cup for scaling purposes.

Therefore, by using the stepper motor at its maximum capacity, it is possible to pump the fluid at the flow rate of $23.75[\mathrm{ml} / \mathrm{min}]$ which is quite higher than the peristaltic micropumps that can be found in literature [16]. However, with respect to the backflows and inefficiencies in terms of trapping the fluid mass, the efficiency of the peristaltic pumps are prone to decrease down to 0.5 . Even in this case, the volumetric flow rate capacity of $11.7[\mathrm{ml} / \mathrm{min}]$ will be sufficiently high for our inertial cell separation application. Our work on the characterization of micropump is still in progress. As a continuation for this research, we want to test our micropump in cell separation experiments, and we also would like to compare the separation efficiencies of the syringe pump and our peristaltic pump. Furthermore, viability of the cells that pass through the micropump channel should be another consideration. One should bear in mind that the squeezing and dragging motion of the peristaltic pump may affect the viability of the cells. If it really happens, this design would not be an ideal solution for the future LOC devices for inertial separation. Our work on these issues are currently in progress.

\section{Conclusions}

An integrated peristaltic micropump mechanism which is capable of supplying fluid flow at $\mathrm{ml} / \mathrm{min}$ scale is designed, optimized, and fabricated. The portable final product is directly integrated to a passive cell separation microchannel which is previously developed by the authors, in which biological cell separation and enrichment tasks can be done. Together with the inlet and outlet tanks, actuator mechanism, programmable LCD screen, tubings, integrated power source, the micropump system can be considered as a self-contained $\mu$-TAS device with a low fabrication cost.

\section{References}

1. D J Laser and J G Santiago. ."A review of micropumps," J. Micromech. Microeng. 14, R35-R64, 2004.

2. Nan-Chyuan Tsai, Chung-Yang Sue. "Review of MEMS-based drug delivery and dosing systems," Sensors and Actuators A 134, 555-564, 2007.

3. D. Xu, L. Wang, G. Ding, Y. Zhou, A. Yu, B. Cai. "Characteristics and fabrication of $\mathrm{NiTi} / \mathrm{Si}$ 
diaphragm micropump," Sensors and Actuators A: Phys. 93, 87-92, 2001.

4. M. Koch, N. Harris, A.G.R. Evans, N.M. White, A. Brunnschweiler. "A novel micromachined pump based on thick-film piezoelectric actuation," Sensors and Actuators A: Phys. 70, 98-103, 1998.

5. S. Guo, T. Fukuda. "Development of the micro pump using ICPF actua- tor," Proceedings of IEEE International Conference on Robotics and Automation, vol. 1, pp. 266-271, 1997.

6. Insoo Leea, Pyohwan Hong, Chanseob Choa, Byeungleul Leec, Kyunghan Chund, Bonghwan Kimd. "Four-electrode micropump with peristaltic motion," Sensors and Actuators. 245, 19-25, 2016.

7. A.V. Lemoff, A.P. Lee. "An AC magnetohydrodynamic micropump," Sensors and Actuators B: Chem., 63, 178-185, 2000.

8. Zhou, J., Papautsky I. "Fundamentals of inertial focusing in microchannels, " Lab on a Chip, 13, 1121-1132, 2013.

9. G. Shuxiang, T. Fukuda. "SMA actuator based novel type of micropump for biomedical application, Proceedings of IEEE International Conference, vol. 2, pp. 1616-1621, 2004.
10. Yobas L., Tang K., Yong S., and Ong E. K. “A disposable planar peristaltic pump for lab-on-a-chip, " Lab on a Chip, 8, 660-662, 2008.

11. Bekin M., Say U., Ozer, A. “Polimer tabanlı kendinden tahrikli entegre mikroparçacık ayrıştırma sistemleri tasarımı, üretimi ve karakterizasyonu [Turkish]," B.S. Thesis, Dept. of Mechanical Engineering, Istanbul Technical University, Istanbul, Turkey, 2014.

12. Zhang X., Chen Z., Huang Y. "A valve-less peristaltic pumping method," Biomicrofluidics. 9, 014118, 2015.

13. Sonmez U., Jaber S., Trabzon L. " Investigation of superposition in microchannel geometry for inertial particle separation, * Proceedings of SB3C International Conference, Maryland, USA, Vol. 1, pp. 1079-1080, June 2016.

14. Lee et. al. "PDMS Nanoslits without Roof Collapse, " Bull. Korean Chem. Soc., Vol. 30, No. 8, 2009.

15. Huang et al. "Stamp collapse in soft lithography, Langmuir, 21, 8058- 8068, 2005.

16. Du M., Ye X., Wu K., Zhou Z. “A peristaltic micro pump driven by a rotating motor with magnetically attracted steel balls, " Sensors, 9, 2611- 2620, 2009. 